\title{
Terapia de reemplazo de nicotina
}

\author{
MARÍA PAZ CORVALÁN B.*
}

\section{Nicotine replacement therapy}

All commercially available forms of nicotine replacement therapy (NRT) (gum, transdermal patch, nasal spray, inhaler and sublingual tablets / pills) can help to quit smoking successfully. NRT increases the cessation rate by 50 to $70 \%$. The combination of a nicotine patch with a rapid dosage form of NRT is more effective than a single type of NRT. There is no difference in efficacy between NRT and bupropion, the combination of NRT and bupropion is more effective than bupropion alone. The effects are largely independent of the duration of therapy, the intensity of the support provided or the setting in which the NRT was offered. According to Chilean experts, NRT can be used in adolescents, as chewing gum $2 \mathrm{mg}$ adding behavioral therapy. If a pregnant woman expresses a clear desire to use NRT, it is suggested (i) discuss the associated risks and benefits with her, (ii) use it only if the cessation fails with non-pharmacological measures and iii) use the professional approach when deciding whether to offer the prescription of NRT, considering the level of addiction of the pregnant woman and the presence of comorbidities. TRN exists in Chile, but is not currently available in the public health system.

Key words: Tobacco cessation products; nicotine; chewing gum; transdermal patch; risk assessment.

\section{Resumen}

Todas las formas comercialmente disponibles de terapia de reemplazo de nicotina (TRN) (chicles, parche transdérmico, aerosol nasal, inhalador y tabletas sublinguales / lozenges) pueden ayudar a de dejar de fumar con éxito. TRN aumenta la tasa cesación en un 50 a 70\%. La combinación de un parche de nicotina con una forma de administración rápida de TRN es más eficaz que un solo tipo de NRT. No hay diferencia en la eficacia entre TRN y bupropión, la combinación de NRT y bupropión es más efectiva que bupropión solamente. Los efectos de TRN son en gran parte independientes de la duración de la terapia, la intensidad del apoyo prestado o lugar en el que se ofreció. Según expertos chilenos, se puede usar TRN en adolescentes, en forma de chicles de $2 \mathrm{mg}$ asociado a terapia conductual. Si una embarazada expresa un claro deseo de recibir TRN, se sugiere (i) discutir con ella los riesgos y beneficios asociados, (ii) utilizarla sólo si falla la cesación con medidas no farmacológicas y (iii) utilizar el criterio profesional al decidir si ofrecer la prescripción de TRN, tomando en cuenta el nivel de adicción de la embarazada y la presencia de comorbilidades. Estos fármacos existen en Chile, pero no están hasta el momento disponibles en el sistema público de salud.

Palabras clave: Productos para dejar de fumar; nicotina; chicles; parches; evaluación de riesgos.

La terapia de remplazo de nicotina (TRN) fue el primer tratamiento para la cesación del tabaquismo disponible y aprobado por la FDA (Food and Drug Administration, USA) y es el que tiene más tiempo y estudios en la historia de terapias para la adicción a la nicotina.

El objetivo de la TRN es reemplazar, temporalmente, la nicotina de los cigarrillos y reducir la motivación para fumar y los síntomas de abstinencia, facilitando así la transición del tabaquis-

* Programa de Tabaquismo Centro Médico Fundación del Banco Estado.

Coordinadora Comisión Tabaco, Sociedad Chilena de Enfermedades Respiratorias. 
mo a la abstinencia completa.

Un estudio Cochrane de $2013^{1}$, evidenció que todas las formas comercialmente disponibles de TRN (chicle, parche transdérmico, aerosol nasal, inhalador y tabletas sublinguales/lozenges) aumentan las posibilidades de dejar de fumar con éxito. Se identificaron 150 ensayos; 117 con más de 50.000 participantes, los que contribuyeron a la comparación primaria entre cualquier tipo de NRT y un grupo de control placebo. La razón de riesgo (RR) de abstinencia para cualquier forma de NRT con respecto al control fue de 1,60 (95\% intervalo de confianza [IC] 1,53 a 1,68). En Chile se encuentran disponibles sólo chicles (los parches estarán disponibles en 2018), los RR combinados para cada tipo fueron 1,49 (IC del 95\%: 1,40 a 1,60, 55 ensayos) para el chicle de nicotina y 1,64 (IC del 95\%: 1,52 a 1,78, 43 ensayos) para el parche de nicotina. TRN aumenta la tasa cesación en un 50 a $70 \%$.

La combinación del parche de nicotina con una forma de administración rápida de NRT fue más eficaz que un solo tipo de NRT (RR 1,34, IC del 95\%: 1,18 a 1,51, 9 ensayos). El efecto del chicle es rápido, lo que permite su uso en control de craving en cambio los parches de nicotina mantienen un nivel estable de nicotina en sangre por 16 a $24 \mathrm{~h}$ atenuando el síndrome de privación, disminuyendo consumo y refuerzo posterior.

Los efectos fueron en gran parte independientes de la duración de la terapia, la intensidad del apoyo prestado o el lugar en el que se ofreció TRN. El efecto fue similar en un pequeño grupo de estudios que uso de TRN obtenido sin receta médica.

Para determinar si TRN es más o menos exitosa en comparación con otras farmacoterapias, cinco estudios compraron directamente TRN con bupropión; no hubo diferencia en la eficacia (RR 1,01; IC del 95\%: 0,87 a 1,18). La combinación de NRT y bupropión fue más efectiva que la indicación de bupropión solamente (RR 1,24; IC del 95\%: 1,06 a 1,45, 4 ensayos).

En 2016 se publicó el estudio EAGLES ${ }^{2}$, ensayo doble ciego, controlado contra placebo, multicéntrico abarcando 16 países, entre ellos Chile, un total de 8.144 participantes fueron asignados a recibir las terapias de vareniclina, bupropión, parche de nicotina o placebo en una cohorte con enfermedad de base en 4.116 pacientes con enfermedad psiquiátrica controlada por tres meses al menos y en 4.028 pacientes sin compromiso psiquiátrico. Este estudio no mostró un aumento significativo en los eventos neuropsiquiátricos adversos atribuibles a vareniclina o bupropión en relación con el parche de nicotina o el placebo.
Según expertos chilenos, se necesita usar TRN en adolescentes ${ }^{3,4,5,6}$, en forma de chicles de $2 \mathrm{mg}$ (un cigarrillo es igual a $1 \mathrm{mg}$ de nicotina) junto a terapia cognitiva conductual, si el adolescente se encuentra motivado (quiere dejar de fumar de aquí a un mes), si es adicto (fuma más de 10 cigarrillos o fuma el primer cigarrillo la primera media hora desde que se levanta), y si el adolescente estima que le es útil. Si una embarazada expresa un claro deseo de recibir terapia de reemplazo de nicotina (TRN) $)^{3,6}$, se sugiere (i) discutir con ella los riesgos y beneficios asociados, (ii) utilizarla sólo si falla la cesación con medidas no farmacológicas y (iii) utilizar el criterio profesional al decidir si ofrecer la prescripción, iiii) Si bien no existe consenso en el esquema terapéutico a usar en embarazadas, expertos internacionales sugieren lo siguiente:

Partir con chicles de nicotina de $2 \mathrm{mg}$. Si esto no funciona considerar el uso de parches de nicotina de $24 \mathrm{~h}$ de duración que se colocan en la mañana y se retiran en la noche. Si la paciente fuma menos de 10 cigarrillos al día usar 1 parche al día de 7 o $14 \mathrm{mg}$. Si fuma 10 o más cigarrillos al día partir con 1 parche al día de $14 \mathrm{mg}$. Evaluar cada 2 semanas y considerar la disminución progresiva de los parches de 14 a $7 \mathrm{mg}$. Usar por un máximo de 2 meses. Notar que solo se justifica mantener los parches si la paciente se mantiene sin fumar.

Basado en esquema terapéutico usado en The Mayo Clinic Dependence Center, USA y Division of Primary Care, University of Nottingham, England.

En la actualidad los chicles de nicotina existen en Chile pero no están disponibles en el sistema público.

\section{Farmacoterapia}

Presentación: chicles de 2 y 4 mg, parches de 7, 14 y $21 \mathrm{mg}$.

Efecto secundario: palpitaciones.

Contraindicaciones: pacientes inestables hemodinámicamente o con arritmias severas.

Asociación de fármacos: pueden ser usadas como terapia única, asociar entre ellas dos formulaciones diferentes (acción rápido y lento) o asociadas a otros fármacos como bupropión o vareniclina.

Prescripción: La TRN se utiliza a partir del Día D (fecha prefijada de inicio del abandono total al tabaco). Técnica de utilización: Chicles; el usuario debe morder, ligeramente el chicle, justo lo suficiente para permitir que la nicotina sea liberada (sabor picante) y se absorba en la 
mucosa bucal (esto demora aproximadamente 10 $\mathrm{min}$ ), esto se logra cuando el chicle se "descansa" en el interior de la mejilla a lo largo de la línea de la encía. El proceso se debe realizar un total de tres veces ya que la duración del chicle es de media hora, se recomienda 3 a 8 chicles al día (1 cigarrillo es igual a $1 \mathrm{mg}$ de nicotina).

Parches: se colocan en zonas accesibles rotando diariamente los sitios tales como hombros, antebrazo, dorso, muslo para evitar dermatitis en el sitio de aplicación. La dosis se titula de acuerdo con el nivel de adicción (para los que fuman más de 15 cigarrillos/día o prenden su primer cigarrillo dentro de la primera hora del día recomendar de $21 \mathrm{mg} /$ día) y se disminuye cada 2 semanas no recomendándose tiempos superiores a las 8 semanas.

En suma, TRN es la más antigua y más estudiada terapia de primera línea para dejar de fumar, disponible actualmente en Chile en forma de chicles que requieren una técnica especial de uso, con muy pocos efectos colaterales y contraindicaciones que permite la combinación de fármacos, sus efectos parecen ser independientes de la intensidad del apoyo prestado y puede ser usado en pacientes con patología psiquiátrica que se encuentra estabilizada por un plazo mayor a tres meses, en adolescentes y embarazadas.

\section{Bibliografía}

1.- CAHILL K, STEVENS S, PERERA R, LANCASTER T. Pharmacological interventions for smoking cessation: an overview and network meta-analysis. Cochrane Database Syst Rev 2013; 5: CD009329.

2. HARTMANN-BOYCE J, STEAD LF, CAHILL K, LANCASTER T. Efficacy of interventions to combat tobacco addiction: Cochrane update of 2013 reviews. Addiction 2014; 109 (9): 1414-25. doi:10.1111/ add. 12633

3.- TOB-g. Tobacco Cessation Guidelines for High Risk Population. http://www.tri.ie/uploads/5/2/7/3/52736649/ tob-g-book-digital-version.pdf (accedido en agosto 2017).

4.- ZWAR N, RICHMOND R, BORLAND R, PETERS M, LITTJ, BELL J, et al. Supporting Smoking Cessation:a Guide for Health Professionals. Melbourne:The Royal Australian College of General Practitioners2011 (updated 2014).

5.- Recommendations of the New Zealand Guidelines for Helping People to Stop Smoking. Wellington: Ministry of Health 2014.

6.- National Institute for Healthcare Excellence (NICE). Smoking: stopping in pregnancy and after childbirth. Public Health Guideline [PH26] (2010). Disponible en: https://www.nice.org.uk/guidance/ph26 (consultado el 19/09/2017). 